\title{
Microprocessor System for Electrical Properties Measuring of Porous Silicon Based Sensors
}

\author{
Petro Parandiy, Nazar Pelypets, Vasyl Rabyk \\ Faculty of Electronics and Computer Technologies \\ Ivan Franko National University of Lviv \\ Lviv, Ukraine
}

\author{
Victor Goshovskiy \\ SR\&PE "Termoprylad - 3" \\ PrJSC NPO “Termoprylad” named after V. Lah \\ Lviv, Ukraine
}

\begin{abstract}
A testing microprocessor system for the study of sensors electric properties based on porous silicon has been developed. The microprocessor system structure, technical characteristics and operation modes are considered. The description of the software for sensors the current-voltage and capacity-voltage characteristic measurements, the results of their experimental research are described.
\end{abstract}

Index Terms - porous silicon; sensor; microprocessing system; current-voltage characteristic; capasitance-voltage characteristic

\section{INTRODUCTION}

The issues of the practical implementation of sensor systems with unique capabilities on porous silicon (PS) are actively discussed in the scientific literature [1,2]. System research of the physical properties of the PS undeniably confirms its high sensitivity (even ultrasensitivity) for a wide class of registered different physical values (temperature, humidity, light radiation, molecular-adsorption interaction, etc.), which opens wide prospects for its use in sensor. At the same time, attempts to create sensory elements based on the PS, which meet the necessary certification requirements for the sensors, encounter significant difficulties.

The porous silicon (PS) is predisposing the attention of researchers mostly due to its luminescent properties [3]. That is, since as in the layers of the PS was detected photoluminescence (PL) with a high quantum efficient at room temperature, this material became the object of intense both fundamental and applied research. Such a widely broad study of various properties of the PS has opened prospects for its numerous alternative applications in areas such as solar cells, biotechnology, sensors [4].

Therefore, the main goal of investigations is the development of sensors on the PS base with the most selectivity established on the current-voltage characteristics (I$\mathrm{V})$ and the capacitance-voltage characteristics (C-V) researching, as well as the method which allows to solve the problem of measurement results interpretation on the base of microprocessor and information technologies and test the new technology of creating real prototypes of PS based sensory systems.
There are exists high quality electronic instrument for measuring and analysing the characteristics of semiconductor structures such as, 4156A Precision Semiconductor Parameter Analysis Agilent [5]. This one instrument allows to perform both high precision characteristic measurement of investigated structures and the EasyEXPERT software package allows to easily transfer the obtaining results to a PC for further data processing. The cost of such complicated instrument is high. Therefore, the main goal of this work was to develop an inexpensive microprocessor system for porous silicon base structure current-voltage and capacity-voltage characteristics investigations, as well as semiconductors (pn-transitions, MOS-structures) and its hardware applying.

\section{MICROPROCESSING SYSTEM HARDWARE}

The microprocessor system (MS) is designed for measuring sensors current-voltage and capacity-voltage characteristics (I$\mathrm{V}$ and $\mathrm{C}-\mathrm{V}$ ) based on porous silicon and semiconductor nanostructures. The functional scheme of the microprocessor system is shown in Fig. 1.

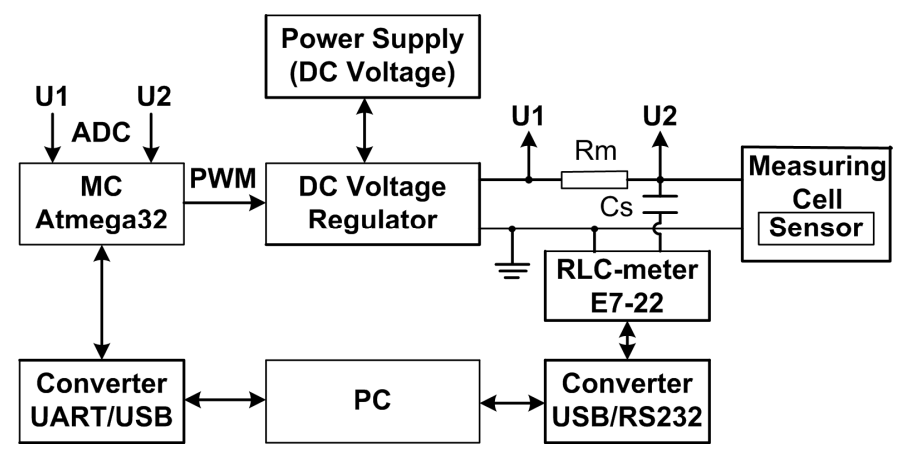

Fig. 1. MS functional scheme of semiconductor nanostructures I-V and C$\mathrm{V}$ characteristics measurement

MC contains next blocks: power supply unit, DC voltage regulator, control and measurement module, RLC-meter E7-22, UART / USB and RS232 / USB converters, measuring cell with researched structure, PC.

The output voltage from the power supply income to the DC voltage regulator unit. The voltage regulator principal scheme is presented in Fig. 2. At the input of RC-circuit $(\mathrm{R} 3 \mathrm{C} 1 \mathrm{R} 4 \mathrm{C} 2)$ the regulator receives a PWM signal of a certain 
duty cycle, which is formed by the Atmega32 microcontroller. This signal charges the capacitors $\mathrm{C} 1$ and $\mathrm{C} 2$ to a certain voltage, the value of which is determined by the amplitude of the PWM signal (for Atmega32 - $5 \mathrm{~V}$ ). Depending on the length of the PWM signal, the voltage on the capacitors will vary from $0 \mathrm{~V}$ to $5 \mathrm{~V}$. Then this voltage is incoming to the noninverting input of the operational amplifier U1: B (TLC2252), where multiplied by the magnitude of the gain $\mathrm{K}=(1+\mathrm{R} 6 / \mathrm{R} 7)$. The output of the amplifier is connected to the transistor VT1 (BC547) base for its control. The output of the VT1 transistor emitter is connected to the measuring resistor R11, the second output of which is connected to the K1 (Out) relay contact. The winding of this relay is controlled by the Atmega32 microcontroller (REL controller input) via the transistor key VT2 (BC847). If the input REL is the logical level "0", then the output OUT_- is connected to the "ground", and the output OUT_+ from Out. If the input REL level is the logical "1", then the output OUT + is connected to the "ground", and the output OUT_- from Out.

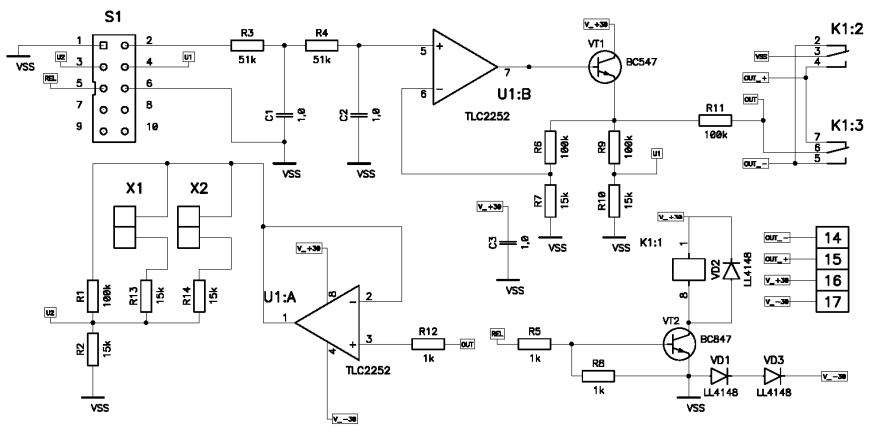

Fig. 2. Regulator DC power supply principal scheme

The OUT + and OUT_- terminals have connected to a measuring cell with the investigated structure. This allows studying the I-V-and C-V dependencies for both positive and negative voltages with unipolar power supply.

Outputs $\mathrm{U} 1$ and $\mathrm{U} 2$ are connected to ADC inputs of Atmega32 microcontroller. Since the voltage regulated by transistor VT1 can significantly exceed ADC reference voltage, the voltage $\mathrm{U} 1$ from the resistive divider R9R10 is applied to ADC input. Another measurement voltage OUT is applied to the non-inverting input of the U1:A operating amplifier, which acts as an emitter follower amplifier. Its output is connected to a resistive divider R2R1R13R14, whose fission factor can be changed by the shorter jumpers X1 and X2. From the output of the divider, the voltage $\mathrm{U} 2$ is incoming to another ADC input.

The control and measurement module is realized on the Atmega32 microcontroller base. The main functions of this module are: generation of PWM signals with given duty cycle, voltage measurement $\mathrm{U} 1$ and $\mathrm{U} 2$, interaction with PC through UART/USB converter for receiving commands and data from $\mathrm{PC}$ and transfer of measurement results to PC.

Atmega32 is an 8-bit low-power AVR microcontroller [6]. Its peripherals include, in particular, two 8-bit timers/counters, one 16-bit timer/counter, 4 PWM generators, 8-channel 10-bit ADC, programmable USART. Its resources are sufficient to implement the operating modes of the microprocessor system.
The capacitance measurement of investigated structures at different bias voltage is performed using RLC-meter E7-22 [7], which RS232 output is connected through RS232/USB converter to the PC. Investigated structure through a $C_{S}$ split capacitor is connected to RLC-meter input. RLC-meter E7-22 allows to measure alternating current at $120 \mathrm{~Hz}$ or $1 \mathrm{kHz}$. The main error of active resistance measuring is $0.5 \%$, and the inductance L and capacity $\mathrm{C}-0.7 \%$ [7]. The smallest range of capacitance measurements is $2000 \mathrm{pF}$, and the largest one is 20 $\mathrm{mF}[7]$.

\section{MiCROPROCESSING SYSTEM SOFTWARE}

The software of the microprocessor system for I-V and C-V characteristics measuring of the investigated structures consists of the software for the control and measurement module implemented on the Atmega32 microcontroller and the main device control program for PC. The software of the system module realizes the algorithm of reference voltage regulating, measures the voltage on the measuring resistance $R_{m}$, transmits the measurement data to the computer and accepts data and commands from PC.

The algorithm for measuring the $\mathrm{I}-\mathrm{V}$ and $\mathrm{C}-\mathrm{V}$ characteristics cconsist in automatically voltage increase at the DC regulator output from the specified value of the voltage minU1 to the value maxU1 with the step $\Delta U 1$. The voltage change range $\mathrm{U} 1$ is $0 \ldots+16 \mathrm{~V}$ due to scheme realization (Fig. 2) on TLC2252 and $-0 \ldots+30 \mathrm{~V}$ when using the LM 258 operating amplifier. The minimum voltage change step is $0.025 \mathrm{~V}$.

Each of the dependencies measurement cycle includes the following procedures: - setting voltage at the constant voltage $\mathrm{U} 1_{\mathrm{t}}$ regulator output; - $1 \mathrm{~s}$ delay time before installing voltage $\mathrm{U} 1_{\mathrm{t}}$; - multiple measurement of $\mathrm{U} 1_{\mathrm{t}}$ and $\mathrm{U} 2_{\mathrm{t}}$ voltages and capacitance $C_{t}$ (time delay between each measuring $0.1 \mathrm{~s}$ ); averaging of the measured values $\mathrm{U} 1_{(\mathrm{m}) \mathrm{t}}, \mathrm{U} 2_{(\mathrm{m}) \mathrm{t}}, \mathrm{C}_{(\mathrm{m}) \mathrm{t}}$ and their transfer to the PC.

The main program algorithm and software are implemented in the C\# programming language in the Visual Studio 2017 environment. It allows you to establish a connection between the $\mathrm{PC}$ and the microprocessor system; configure the virtual com-port settings; transfer control commands to the microprocessor system and RLC-meter E7-22; receive the measurement results from them; store the received data in files and build graphics. The interface of the main program is presented in Fig. 3. It consists of a menu bar (File, Setup, Help) and several areas, numbered in Fig. 3.

In the 1st area (Measurement Setup) parameters are set: the measured characteristic (VA Curves or VF Curves); Input voltage range - minimum, maximum value, step; Number of measurement. All data for these parameters is selected from their windows. When you click the "Upload to Hardware" button, the values of all parameters will be transmitted through the virtual Com-port to the microcontroller. This requires that the Com-port be opened. To configure the parameters of the RS-232 interface, go to the Setup menu and in the window that opens (Fig. 4), select the RS232 submenu. 


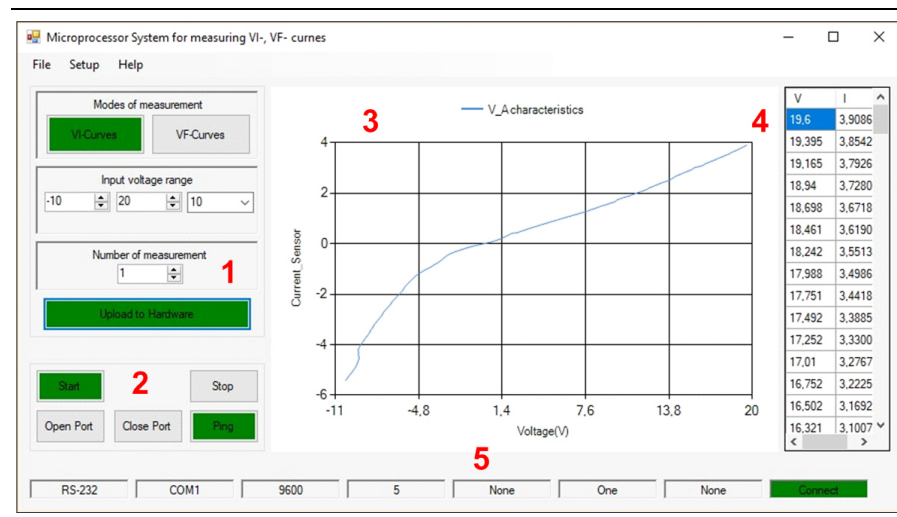

Fig. 3. View of the main program interface

The ReScan button allows you to scan all ports connected to the PC. When the Connect button is pressed, the selected Com- port is opened with the specified parameters.

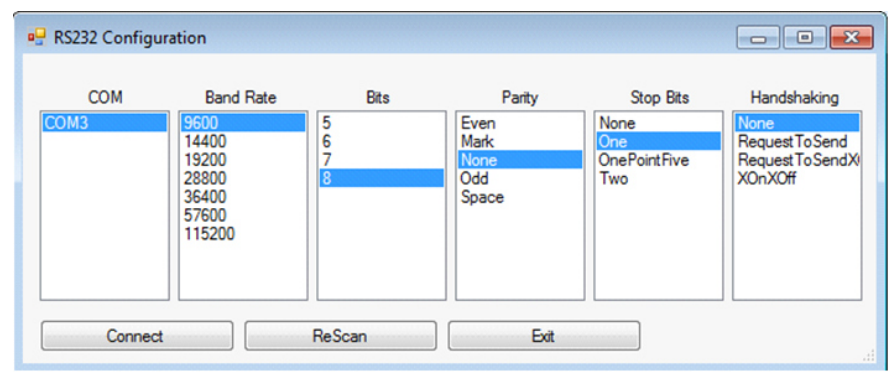

Fig. 4. RS232 interface settings configuration window

In the 2nd area there are the Start, Stop, Open Port, Close Port and Ping buttons. To activate the start of measuring it is necessary to click on the Start button of the program interface. You can stop the measurement by pressing the Stop button. If the RS-232 interface is configured, opening and closing the Com-port can be done using the Open Port, Close Port buttons, which are located on the program interface form. You do not need to go into the Setup $\rightarrow$ RS232 menu. You can check the connection of the microprocessor system with the computer by clicking on the Ping button.

The visualization window (area 3) allows displaying the measured or recorded data in the graphs mode I-V or $\mathrm{C}-\mathrm{V}$ characteristics. In the 4 area are displayed measured values or recorded in the file. Fig. 3 shows the graph of measured I-V characteristics and their numerical values for PS-nSi (n-type silicon sulfur doped substrate marked KES-0.01.

In the 5 area, RS-232 interface parameters are displayed, which are valid at the present time (the last parameters with which the program worked is stored).

The control and measurement module software is realized in the Code Vision AVR environment in $\mathrm{C}$ programming language. The program consists of a main program and two timer TIMER0 subprogram interrupt processing and data reception on USART0 serial port. This measurement is implemented in the TIMER0 timer interrupt subprogram. The PWM generator is based on the timer TIMER1.
IV. Porous Silicon Testing SEnsor PreParation

Single crystal silicon wafers obtained by zone melting growth with crystallographic orientation (100) and (111) were used to producing PS samples in electrochemical etching. The thickness of the substrate was $400 \mu \mathrm{m}$ and a diameter of 76 or $100 \mathrm{~mm}$ respectively. PS layers grown directly on silicon substrates p- and n- type doping with a resistivity of 0.01 to 12 $\Omega \cdot \mathrm{cm}$. Both the surface of single-crystal wafers was mirror polished.

In order to remove the native oxide layer on the samples, they were etched in dilute $(10 \%)$ hydrofluoric acid (HF). A Teflon chamber is the simplest electrolytic cell to form a PS. Anode is a monocrystalline silicon plate and cathode - grid of platinum or other resistant to hydrofluoric acid conductive material. As the electrolyte during anodization is typically used ethanol solution of hydrofluoric acid with the ratio of components $\mathrm{C}_{2} \mathrm{H}_{5} \mathrm{OH}: \mathrm{HF}=1: 1$. The sample were mounted in the etching cell in such a way that the current required for etching process could complete the electrical circuit between the irradiated surface and the bottom surface of the $\mathrm{Si}$ substrate. A 100W UV lamp was used as the photon beam source for preparing a PS specimen with n-type conductivity with the aim of positive charge carriers generation. The irradiation time for the etching was taken to be during the all etching process. The choice of electrochemically formation time depends on the electrical properties of the prepared samples. After the etching process, the samples were rinsed with ethanol and washed by distilled water.

For investigation the set of PS electrochemically etched samples were selected: PS-pSi (p-type silicon boron doped substrate marked KDB-10 j = $5 \mathrm{~mA} / \mathrm{cm}^{2}, \mathrm{t}=20 \mathrm{~min}$ ), PS-nSi (n-type silicon sulfur doped substrate marked KES-0.01, $\mathrm{j}=35$ $\mathrm{mA} / \mathrm{cm}^{2}, \mathrm{t}=50 \mathrm{~min}$ ).

Etched substrates were separated on the single samples of $1 \mathrm{~cm}^{2}$ square approximately. Contact to the porous layer about $2 \mathrm{~mm}$ in diameter was obtained by electrically conductive graphite varnish or glue.

Fig. 5 shows the surface of the PS on the substrate of n-type conductivity KES-0.01 (PS-nSi), obtained with a scanning electron microscope REEMA-102-02.

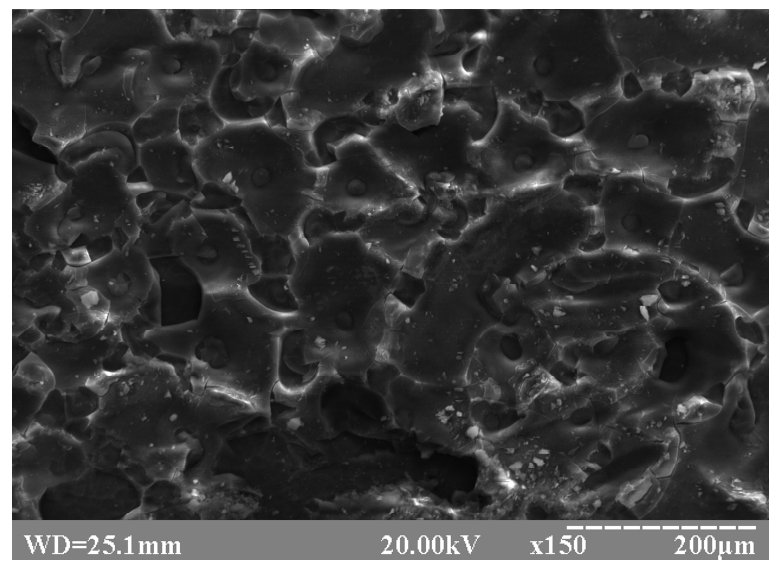

Fig. 5. a) PS-nSi scanning electron surface image, b) PS-nSi cross-section scanning electron surface image. 
Unlike the flat crystalline silicon surface upside of PS is matte and dark and there is clearly sponge-like structured surface. Color surface after electrochemical etching is dark Cross-section scan electron image confirm that the PS layer has sponge-shaped disordered etched pores.

\section{CONCLUSION}

The microprocessor system functional scheme for I-V and $\mathrm{C}-\mathrm{V}$ characteristic measurements (DC power supply, voltage regulator, control and measurement module, RLC-meter E7-22, measured cell with sensor) of sensor based on porous silicon and semiconductor nanostructures is developed. The functional scheme is constructed in the form of a sample-layout.

An algorithm for sensors electrical properties measuring has been developed and software has been implemented. That allows to measure the $\mathrm{I}-\mathrm{V}$ and $\mathrm{C}-\mathrm{V}$ characteristics of these sensors, visualize their dependencies, and store the measurement results in the files.

Microprocessor system testing using the PS-nSi sensor and comparing the results with the results of constant voltage and current measurements in manual mode using Keithley 2100 devices was provided. The results of the comparison allow us to conclude that the implemented modules of the microprocessor system for sensors I-V- and C-V characteristics testing can be used in the development of portable environmental monitoring devices, to study porous silicon sensors using them.

\section{REFERENCES}

[1] Farid A.Harraz, Porous silicon chemical sensors and biosensors: A review, Sensors and Actuators B: Chemical, Volume 202, 31 October 2014, pp. 897-912

[2] Igor A. Levitsky, "Porous Silicon Structures as Optical Gas Sensors" Sensors, (Basel). 2015 Aug; 15(8), pp. 19968-19991

[3] Canham L.T. Appl. Phys. Lett., vol. 57, 1990, p.1046-1048.

[4] C. Baratto, G. Faglia, G. Sberveglieri, "Multiparametric porous silicon sensors," Sensors, 2002, vol. 2, p. 121-126.

[5] Semiconductor Parameter Analyzer. [Web-resource]. Access mode: https://www.keysight.com/upload/cmc_upload/All/04155-90015.pdf

[6] 8-bit Microcontroller with 32KBytes In-System Programmable Flash Atmega32/Atmega321. [Web-resource]. Access mode: http://ww1.microchip.com/downloads/en/DeviceDoc/doc2503.pdf

[7] RLC meter. [Web-resource]. Access moded: http://www.kosmodrom.com.ua/pdf/E7-22.pdf 